\title{
AN INITIAL-VALUE PROBLEM IN SHOCK STABILITY
}

\author{
BY G. W. SWAN (Washington State University)
}

A study of the stability of normal shock waves in fluids with viscosity and heat conduction is presented by Morduchow and Paullay [1]. In dealing with the structure for a continuous weak shock the following equation, their (41), is obtained:

$$
\frac{\partial \bar{u}}{\partial t}+\left(u_{s}-\frac{1+\alpha}{2}\right) \Gamma \frac{\partial \bar{u}}{\partial x}+\Gamma u_{s}^{\prime} \bar{u}=\frac{1}{2} \delta \frac{\partial^{2} \bar{u}}{\partial x^{2}},
$$

$(-\infty<x<\infty, t>0)$, where $\bar{u}(x, t)$ is a small perturbation on the steady-state velocity:

$$
u_{s}(x)=\frac{1}{2}\left[1+\alpha-(1-\alpha) \tanh \frac{1}{2} \delta^{-1} \Gamma(1-\alpha) x\right],
$$

and $\alpha, \delta(>0)$ and $\Gamma$ are constants. The boundary conditions are

$$
x \rightarrow-\infty, \quad \bar{u} \rightarrow 0, \quad u_{s} \rightarrow 1, \quad x \rightarrow+\infty, \quad \bar{u} \rightarrow 0, \quad u_{s} \rightarrow \alpha .
$$

In [1] only the nature of the continuous eigenvalue spectrum is investigated. The complete formulation of the above problem requires that the initial form of the perturbation $\bar{u}(x, 0)$ be specified:

$$
\bar{u}(x, 0)=A(x),
$$

say.

The purpose of this note is to illustrate how one can obtain an explicit solution to the initial- and boundary-value problem posed by (1)-(4).

The coefficients of $\bar{u}_{x}$ and $\bar{u}$ in (1) are complicated hyperbolic functions. By introduction of a change of variables it is possible to arrange for these coefficients to be algebraic in nature. This may be achieved as follows. Introduce $X, t$ as the new independent variables, with $X=\left(1-u_{s}\right) /(1-\alpha)$. With $\Omega(X, t)$ denoting $\bar{u}\left(x\left(u_{s}(X)\right), t\right)$ the problem (1)-(4) is now formulated as

$$
\begin{gathered}
\left(X-X^{2}\right)^{2}\left(\partial^{2} \Omega / \partial X^{2}\right)+2\left(X-X^{2}\right) \Omega=k(\partial \Omega / \partial t), \\
\Omega(0, t)=\Omega(1, t)=0, \quad \Omega(X, 0)=F(X),
\end{gathered}
$$

where $k=2 \delta /(1-\alpha)^{2} \Gamma^{2}>0$, and, for convenience, the initial form of the perturbation $A\left(x\left(u_{s}(X)\right)\right)$ is replaced by $F(X)$.

Eq. (5) is linear and this suggests the use of integral transform techniques. Introduce the Laplace transform of $\Omega(X, t)$ :

$$
\Phi(X, p)=\int_{0}^{\infty} \Omega(X, t) \exp (-p t) d t
$$

* Received November 16, 1972. 
Direct application of this transform to (5) gives

$$
\Phi^{\prime \prime}+\left[2\left(X-X^{2}\right)^{-1}+\lambda\left(X-X^{2}\right)^{-2}\right] \Phi=f(X),
$$

where

$$
\lambda=-k p, \Phi=\Phi\left(X,-\lambda k^{-1}\right), \quad f(X)=-k \Omega(X, 0)\left(X-X^{2}\right)^{-2},
$$

and $\Omega(X, 0)$ represents the initial form of the perturbation. Also, since $\Omega(0, t)=$ $\Omega(1, t)=0$,

$$
\Phi\left(0,-\lambda k^{-1}\right)=\Phi\left(1,-\lambda k^{-1}\right)=0 .
$$

Mathematically, here, we have a singular eigenfunction expansion problem. The determination of $\Phi$ and the spectrum of eigenvalues is not trivial.

Let $\varphi(X, \lambda), \psi(X, \lambda)$ be two solutions of the homogeneous equation (namely (8) with $f \equiv 0$ ) such that their Wronskian $W(\varphi, \psi)=1$; then it is straightforward, by differentiation, to show that

$$
\Phi\left(X,-\lambda k^{-1}\right)=\psi(X, \lambda) \int_{0}^{X} \varphi(X, \lambda) f(X) d X+\varphi(X, \lambda) \int_{X}^{1} \psi(X, \lambda) f(X) d X
$$

is the solution of (8). To find $\varphi$ and $\psi$ proceed as follows. Introduce

$$
U=X^{r}(1-X)^{m}(n-X),
$$

where $\tau, m$ and $n$ are as yet undetermined quantities. Consider the homogenoeus equation

$$
L \Phi=0, L \equiv d^{2} / d X^{2}+2\left(X-X^{2}\right)^{-1}+\lambda\left(X-X^{2}\right)^{-2} .
$$

Now

$$
L U=X^{\tau-2}(1-X)^{m-2}\left(P+Q X+R X^{2}+S X^{3}\right),
$$

where

$$
\begin{aligned}
& P=n\left(\tau^{2}-\tau+\lambda\right), \quad Q=-2 n \tau(m-1+\tau)-\tau^{2}-\tau+n+\lambda, \\
& R=2\left(\tau^{2}+\tau m-n\right)+(m-1+\tau)(n \tau+m n+2), \\
& S=(m+2+\tau)(m-1+\tau) .
\end{aligned}
$$

The quantity $S$ can be chosen to be zero if

$$
m=1-\tau,
$$

and for this value of $m, R=2(\tau-n)$, which can be made zero for

$$
n=\tau \text {. }
$$

Also, on using (15) and (16), $Q=-\left(\tau^{2}-\tau+\lambda\right)$ and if $\tau$ is chosen to satisfy

$$
\tau^{2}-\tau+\lambda=0
$$

$P$ and $Q$ are now zero and $L U=0$ with

$$
U=X^{\tau}(1-X)^{1-\tau}(\tau-X) .
$$

However, the coefficient $S$ can also be chosen to be zero for $m=-2-\tau$ and it is readily verified that this choice does not give consistency when the quantities $P, Q$ and $R$ are 
set to zero. Consequently this value of $m$ is dismissed. The solution of (17) is

$$
\tau=\frac{1}{2}-\frac{1}{2} i(4 \lambda-1)^{1 / 2}, \quad \tau_{1}=\frac{1}{2}+\frac{1}{2} i(4 \lambda-1)^{1 / 2} .
$$

Two linearly independent solutions of $L \Phi=0$ are now (18) and

$$
V=X^{\tau_{1}}(1-X)^{1-\tau_{1}}\left(\tau_{1}-X\right)
$$

and, since $\tau_{1}=1-\tau$,

$$
V=X^{1-\tau}(1-X)^{\tau}(1-\tau-X),
$$

with $\tau$ being given by the first equation of (19). Furthermore

$$
W(U, V)=-(1-2 \tau)\left(\tau^{2}-\tau\right)=i \lambda(4 \lambda-1)^{1 / 2},
$$

and hence

$$
\begin{aligned}
& \varphi(X, \lambda)=\left[i \lambda(4 \lambda-1)^{1 / 2}\right]^{-1} X^{\tau}(1-X)^{1-\tau}(\tau-X), \\
& \psi(X, \lambda)=X^{1-\tau}(1-X)^{\tau}(1-\tau-X),
\end{aligned}
$$

with $2 \tau=1-i(4 \lambda-1)^{1 / 2}$, are two linearly independent solutions of $L \Phi=0$ such that $W(\varphi, \psi)=1$.

Finally, substitution of the forms (20), (21) for $\varphi$ and $\psi$ into (11) gives the solution of (8) with boundary conditions (10). Inversion of (7) gives

$$
\Omega(X, t)=-(2 \pi i k)^{-1} \int_{-k c+i \infty}^{-k c-i \infty} \Phi\left(X,-\lambda k^{-1}\right) \exp \left(-\lambda k^{-1} t\right) d \lambda,
$$

where $c$ is a positive constant. Since $k$ is positive, $k c$ is positive. There is a pole of $\Phi$ at $\lambda=0$ and a branch-point singularity at $\lambda=\frac{1}{4}$. The evaluation of (22) is (formally) accomplished by closing the contour in the right-hand half-plane. Let $C_{1}$ be the arc of the quarter circle from $-k c-i \infty$ to $\infty, C_{2}$ be the lower branch from $\infty$ to $\frac{1}{4}+\delta, C_{3}$ be the arc of a small circle, radius $\delta$, surrounding $\lambda=\frac{1}{4}, C_{4}$ be the upper branch from $\frac{1}{4}+\delta$ to $\infty$ and $C_{5}$ be the are of the quarter circle from $\infty$ to $k c+i \infty$. On $C_{1}$ and $C_{5}$, $\lambda=\operatorname{Re}^{i \theta}$, say, and as $R \rightarrow \infty$ the presence of the decaying exponential in the integrand in (22) assures that there are no contributions from $C_{1}$ and $C_{5}$. The residue at $\lambda=0$ is given by

$$
2 \pi i k\left(X-X^{2}\right) \int_{0}^{1}\left(X-X^{2}\right)^{-1} \Omega(X, 0) d X .
$$

On the branch $C_{2}, \lambda=\frac{1}{4}+\mathrm{re}^{2 \pi i}$ and on the branch $C_{4}, \lambda=\frac{1}{4}+r$. Finally, the perturbation (in the limit as $\delta \rightarrow 0$ )

$$
\begin{aligned}
\Omega(X, t)= & \left(X-X^{2}\right) \int_{0}^{1}\left(X-X^{2}\right)^{-1} \Omega(X, 0) d X \\
& +(2 \pi i k)^{-1}\left[-\int_{1 / 4}^{\infty} F(X, r) d r+\int_{1 / 4}^{\infty} G(X, r) d r\right],
\end{aligned}
$$

where $F(X, r), G(X, r)$ are the contributions from $C_{2}$ and $C_{4}$, respectively. After a little 
algebraic manipulation this expression can be cast in the form

$$
\begin{aligned}
\Omega(X, t)= & \left(X-X^{2}\right) \int_{0}^{1}\left(X-X^{2}\right)^{-1} \Omega(X, 0) d X \\
& +(4 \pi)^{-1} \int_{1 / 4}^{\infty}\left[a(X, r) a_{1}(r)+b(X, r) b_{1}(r)\right] \exp \left(-r k^{-1} t\right) d r,
\end{aligned}
$$

where

$$
\begin{gathered}
a(X, r)=X^{1 / 2+i s}(1-X)^{1 / 2-i s}\left(\frac{1}{2}+i s-X\right) s^{-1}\left(\frac{1}{4}+r\right)^{-1}, \\
a_{1}(r)=\int_{0}^{1} X^{-3 / 2-i s}(1-X)^{-3 / 2+i s}\left(\frac{1}{2}-i s-X\right) \Omega(X, 0) d X
\end{gathered}
$$

and $b(X, r), b_{1}(r)$, respectively, are the same as $a(X, r), a_{1}(r)$, respectively, but with $i$ replaced by $-i$; also $s=r^{1 / 2}$.

The first expression on the right of (23) is interpreted as being the neutrally stable mode. It represents a translation of the weak shock structure and does not damp out with time.

\section{REFERENCE}

[1] M. Morduchow and A. J. Paullay, Stability of normal shock waves with viscosity and hcat conduction, Phys. Fluids 14, 323-331 (1971) 